\title{
THE CZECH CONTRIBUTION TO FUTURE LARGE X-RAY ASTRONOMY TELESCOPES: RECENT PROGRESS
}

\author{
René Hudec ${ }^{a, b, *}$, Ladislav Pína ${ }^{c}$, Adolf Inneman $^{d}$, Daniela Černá ${ }^{d}$, \\ Veronika MarŠíkovád, ${ }^{d}$ Vladimír TichÝ ${ }^{e}$, Martin MíkA ${ }^{f}$ \\ a Astronomical Institute, Academy of Sciences of the Czech Republic, CZ-25165 Ondrejov, Czech Republic \\ ${ }^{b}$ Czech Technical University in Prague, Faculty of Electrical Engineering, Technická 2, CZ-16627 Prague, Czech \\ Republic \\ ${ }^{c}$ Czech Technical University in Prague, Faculty of Nuclear Science and Physical Engineering, Břehová 78/7, \\ CZ-11000 Prague, Czech Republic \\ ${ }^{d}$ Rigaku Innovative Technologies Europe s.r.o., Novodvorská 994, CZ-14221 Prague 4, Czech Republic \\ ${ }^{e}$ Czech Technical University in Prague, Faculty of Biomedical Engineering, Nám. Sítná 3105, 272 01 Kladno, \\ Czech Republic \\ f Institute of Chemical Technology, Technická 5, CZ-16628 Prague, Czech Republic \\ * corresponding author: rhudec@asu.cas.cz
}

\begin{abstract}
We briefly review the recent status of the Czech contribution to future space X-ray astronomy missions with emphasis on the development of new technologies and test samples of X-ray mirrors with precise surfaces based on new materials and alternative designs. We report on further investigations and tests of X-ray optical arrangements, such as Kirkpatrick-Baez systems and Multi-Foil Optics.
\end{abstract}

KEYWORDS: X-ray astronomy, X-ray telescopes, X-ray optics.

\section{INTRODUCTION}

This paper gives a brief review of recent efforts in the Czech Republic in the development of novel space Xray imaging optics. The design and development of $\mathrm{X}$ ray optics have a long tradition in the Czech Republic (e.g. Hudec et al., 1991, 1999, 2000, 2001, Inneman et al. 1999, 2000). A range of related technologies have been exploited and investigated over the years, including technologies for future large, light-weight $\mathrm{X}$-ray telescopes.

Future large space X-ray telescopes (such as Athena [16]) require precise, light-weight X-ray optics based on numerous thin reflecting shells. Novel approaches and advanced technologies need to be developed and exploited. In this paper, we refer to Czech efforts focusing on various novel designs, approaches and technologies. In order to achieve sub-arsec angular resolution, the principles of active optics also need to be addressed.

\section{PROGRESS IN SILICON WAFER SHAPING}

Silicon wafers are intended for use as mirrors in the ESA XEUS, IXO and Athena projects. As in the case of XEUS and IXO, the baseline optics for the Athena X-ray telescope design was based on X-Ray High Precision Pore Optics (X-HPO), a technology developed with ESA funding (RD-Opt, RD-HPO), with a view to achieving large effective areas with low mass, reduced telescope length, high stiffness, and a monolithic structure, favoured for handling a thermal environment and for simplifying the alignment process (Bavdaz et al. 2010). In addition, due to the higher packing density and the associated need for shorter mirrors, conical approximation to the Wolter-I geometry becomes possible. X-HPO optics is based on ribbed Si wafers stacked together. To achieve conical approximation, the Si wafers are formed by stacking a large number of plates together using a mandrel. The typical size of the Si wafers is $100 \times 100 \mathrm{~mm}$.

There are also alternative X-ray optics arrangements using Si wafers. In this paper, we refer to the further development of an alternative design of innovative precise X-ray optics based on Si wafers. Our approach is based on two steps, namely:

(1.) developing dedicated $\mathrm{Si}$ wafers with properties optimized for use in space X-ray telescopes;

(2.) precise shaping of the wafers to the optical surfaces.

To achieve nested arrays, stacking is performed after the wafers have been shaped. In this approach, Multi Foil Optics (MFO) is thus created from shaped $\mathrm{Si}$ wafers. For more details on MFO, see Hudec et al. (2005).

This alternative approach does not require the $\mathrm{Si}$ wafers to have a ribbed surface, so problems with transferring any deviation, stress, and/or inaccuracy from one wafer to the neighbouring plates, or even to the whole stacked assembly, will be avoided. However, suitable technologies for precise stacking of optically 

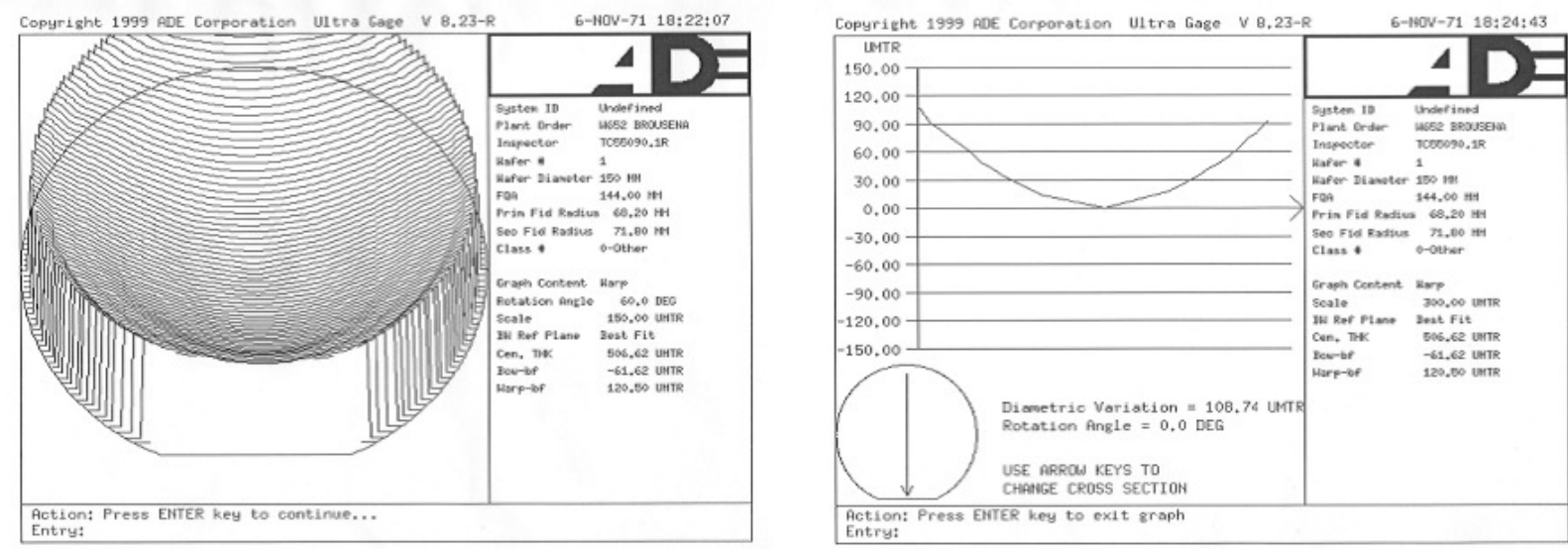

Figure 1. Example of precise silicon wafer shaping by thin layer deposition. After the deposition of poly-Si (thickness $1436 \mathrm{~nm}$ at temperature $\left.615^{\circ} \mathrm{C}\right)$ and for wafer thickness $507 \mu \mathrm{m}$, a warp of $110 \mu \mathrm{m}(R=25.6 \mathrm{~m})$ was achieved. Left: $\mathrm{Si}$ wafer deformation map. Right: Warp profile perpendicular to the facet.

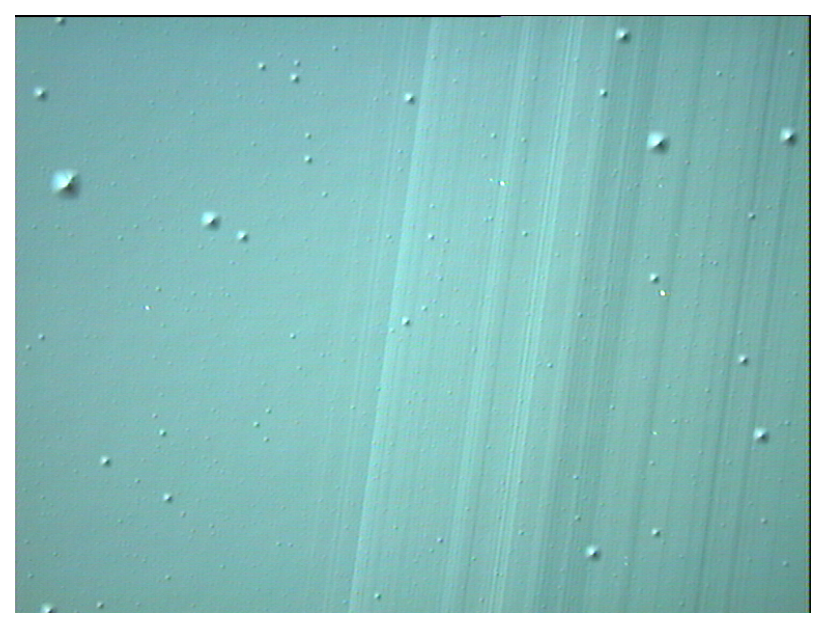

Figure 2. Microphotograph of slip systems on an Si wafer after plastic deformation. This study was aimed at achieving a better understanding of the process of $\mathrm{Si}$ plastic deformation in order to retain the surface quality.

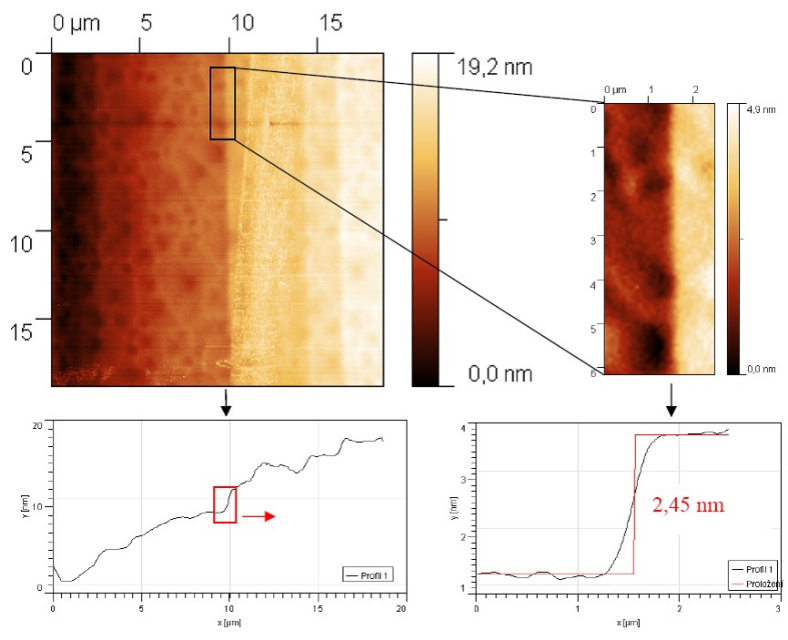

Figure 3. The Si (100) wafer surface morphology after plastic deformation - AFM measurements.

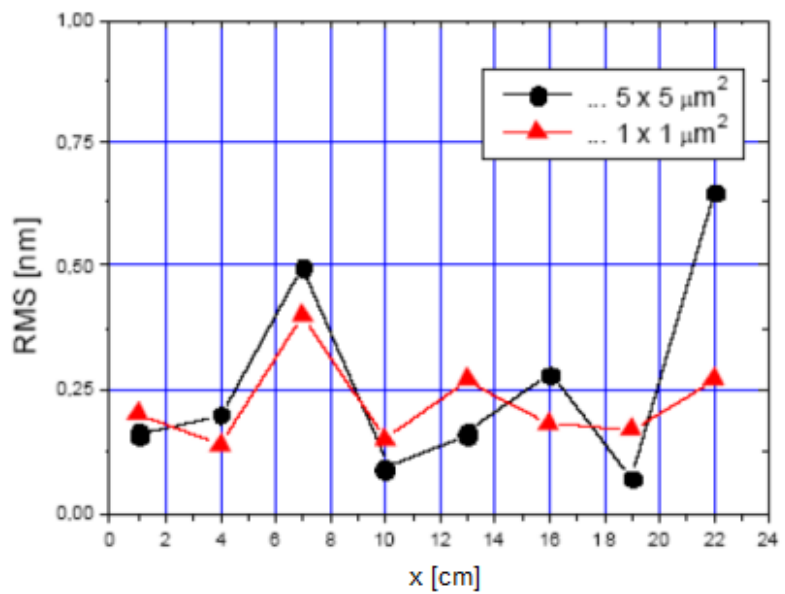

Figure 4. Dependence of RMS on scan size, measured on 8-inch silicon wafers at Brno University of Technology.

formed wafers to a multiple array have yet to be developed.

In order to achieve the very high accuracy required for future large space X-ray telescopes, the parameters of the Si wafers need to be optimized (for application in X-ray optics) at the production stage. For this purpose, we have established and developed a multidisciplinary working group including development specialists from the Si wafer industry with the goal of designing and manufacturing Si wafers with improved parameters (mostly flatness) optimized for application in X-ray telescopes. It should be noted that the manufacture of silicon wafers is a complicated process with numerous technological steps and with many free parameters that can be modified and optimized to achieve optimal performance. This can also be useful for further improving the quality of $\mathrm{X}-\mathrm{HPO}$ optics. As we are dealing with high-quality X-ray imaging, the smoothness of the reflecting surface is important. The standard microroughness of 

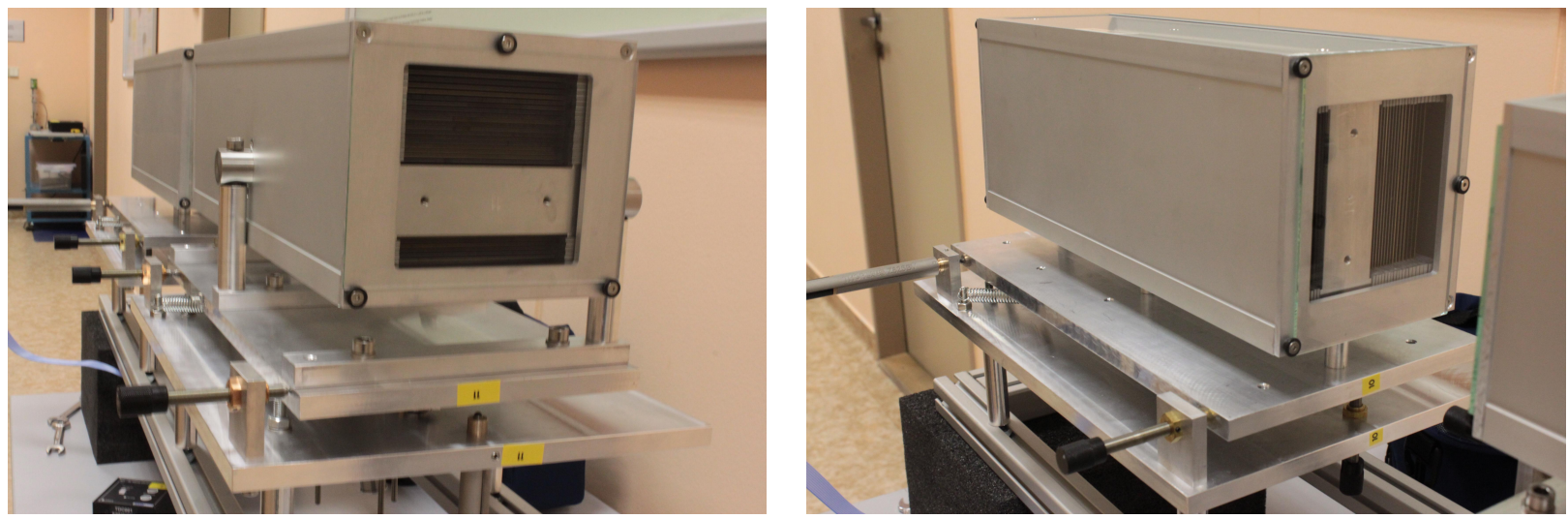

Figure 5. Test K-B modules recently assembled at RITE in Prague. The largest KB module includes 72 silicon mirrors, squared to $100 \times 100 \mathrm{~mm}, 0.525 \mathrm{~mm}$ in thickness, with typical plate separation between 2 and $3 \mathrm{~mm}$.
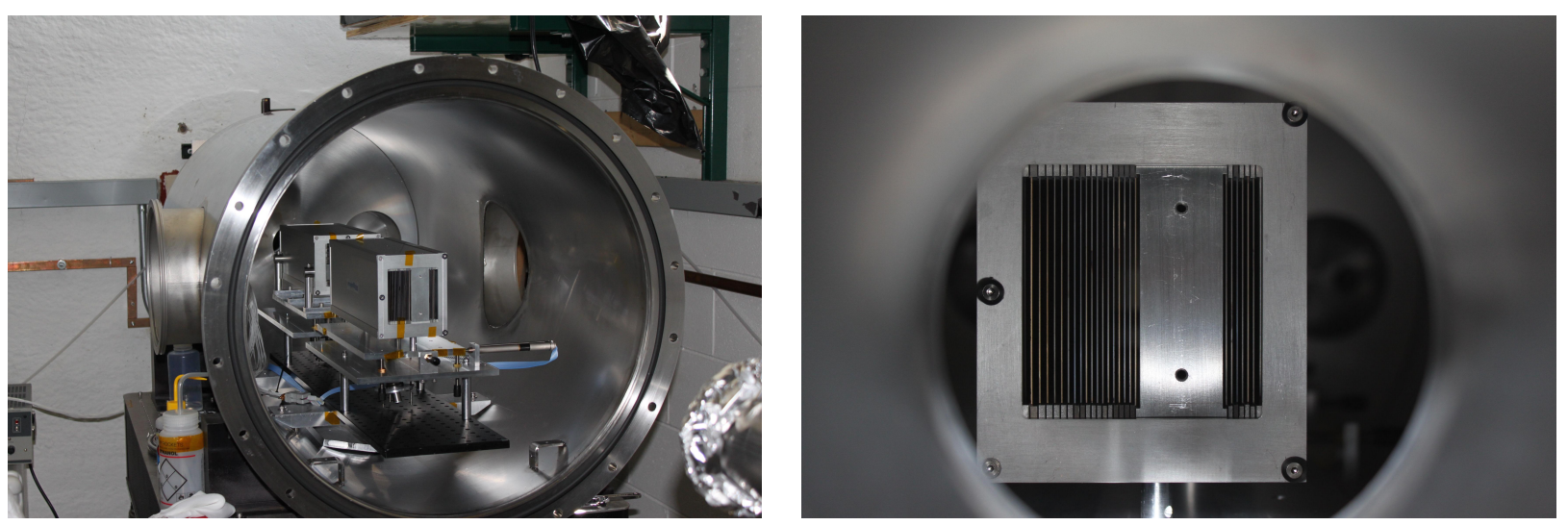

Figure 6. The KB test modules during the tests in spring 2012 at the facility for full aperture X-ray tests at the University of Colorado at Boulder. All tested modules were tested in full aperture (diamater $80 \mathrm{~mm}$ ) focus to focus arrangement $(2 \times 10 \mathrm{~m})$.

commercially available Si wafers (we have used products from ON Semiconductor, Czech Republic) is of the order of $0.1 \mathrm{~nm}$, as confirmed by several independent measurements by various techniques, including the Atomic Force Microscope (AFM). This is related to the method of chemical polishing used in the manufacture of Si wafers. The microroughness of $\mathrm{Si}$ wafers is lower than the microroughness of glass foils and most other alternative mirror materials and substrates.

Below, we present examples of results obtained recently, illustrating current progress in Si wafer shaping for X-ray optics applications. The goal was to achieve improved quality, improving the precision and hence the parameters and performance of the optics.

\section{AdVANCED KiRKPATRICK-BAEZ X-RAY TEST MODULES}

As was mentioned in the previous section, Si wafers are difficult to shape, especially to small radii. To overcome this difficulty, another X-ray optics arrangement can be considered, namely the Kirkpatrick-Baez (KB) system. Then the curvature radii are much larger, of the order of a few $\mathrm{km}$, while the imaging performance is similar. For the same effective area, however, the focal length of the KB system is twice as large as the focal length of the Wolter system. Nevertheless, KB systems offer a promising alternative to the classical Wolter systems in future large space $\mathrm{X}$-ray telescopes.

A very important consideration is the ease (and hence the reduced cost) of constructing highly segmented modules based on multiply nested thin reflecting substrates in comparison with the Wolter design. While, for example, the Wolter design for Athena required the substrates to be precisely formed with curvatures as small as $0.25 \mathrm{~m}$, the alternative $\mathrm{KB}$ arrangement uses only slightly bent mirrors. Hence the feasibility of constructing a KB module with the required 5 arcsec FWHM at an affordable cost is higher than for the Wolter arrangement.

Advanced KB telescopes are based on the Multi Foil Optics (MFO) approach (X-ray grazing incidence imaging optics based on numerous thin reflecting mirrors/foils). The distinction between MFO and other optics using packed or nested mirrors is that MFO is based on numerous very thin substrates (typically less than $0.1 \mathrm{~mm}$ ). Advanced MFO KB test modules 

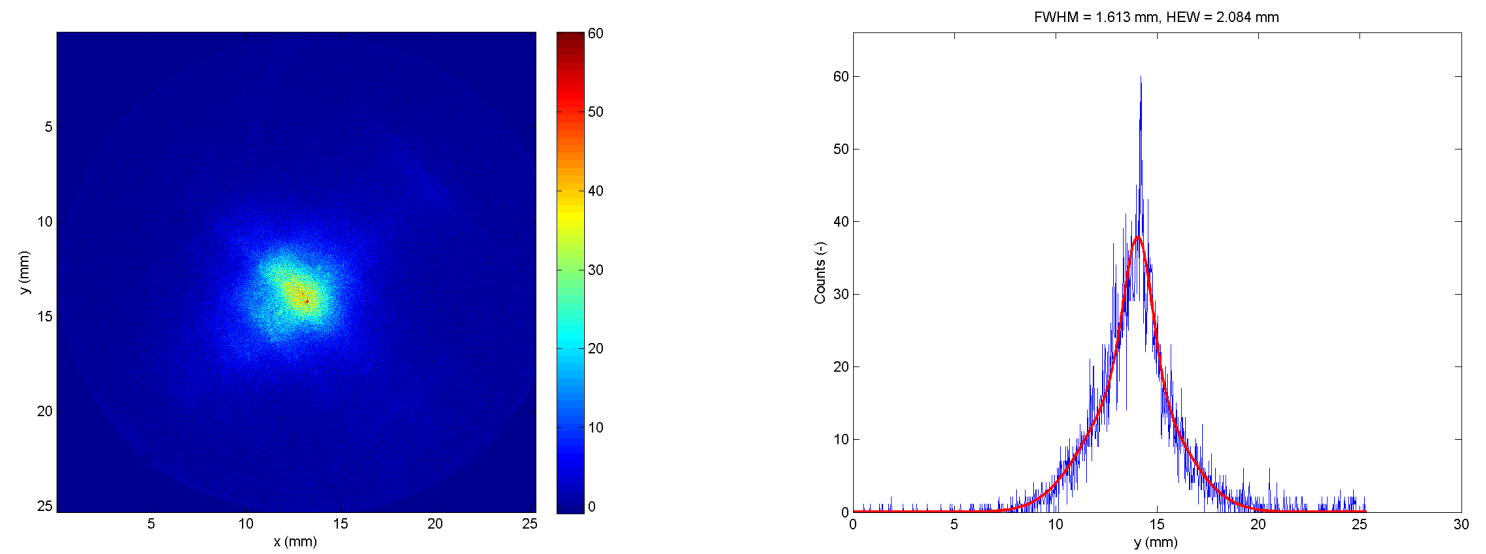

Figure 7. Measurement results of the KB advanced test module with Si mirrors, full aperture tests at $5 \mathrm{keV}$ at the University of Colorado at Boulder. Detector based on MCP, diameter 1 inch. The estimated FWHM of 2D focus (after correction for final focal length) is 11 arcsec, and HEW is 14 arcsec.

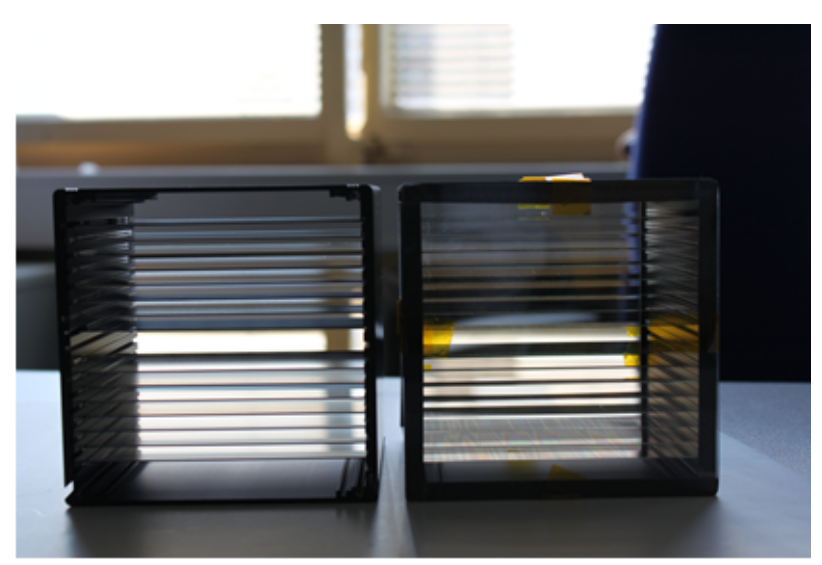

Figure 8. Examples of test modules of X-ray MFO optics recently assembled (spring 2012) at RITE in Prague, with both glass foils and silicon mirrors, prepared for full aperture tests at the University of Colorado in Boulder test facility.

were recently designed and assembled at Rigaku Innovative Technologies Europe (RITE) in Prague, and several modules were tested in full aperture X-ray tests at the test facility of the University of Colorado at Boulder in April and May 2012, with preliminary results of about FWHM 10-20 arcsec for a full stack of a module with Si mirrors at $5 \mathrm{keV}$. In our opinion, the use of the KB design instead of Wolter in future large space X-ray telescopes may help to retain high performance (e.g. effective area) even with a reduced budget.

\section{Multi Foil (MFO) X-Ray Optics}

Novel mirrors for X-ray optics, e.g. thin glass foils and thin silicon wafers, allow various Multi Foil X-ray Optics designs (MFO) to be considered and tested. These systems, with different designs and geometries, may have various applications both in space and in the laboratory.

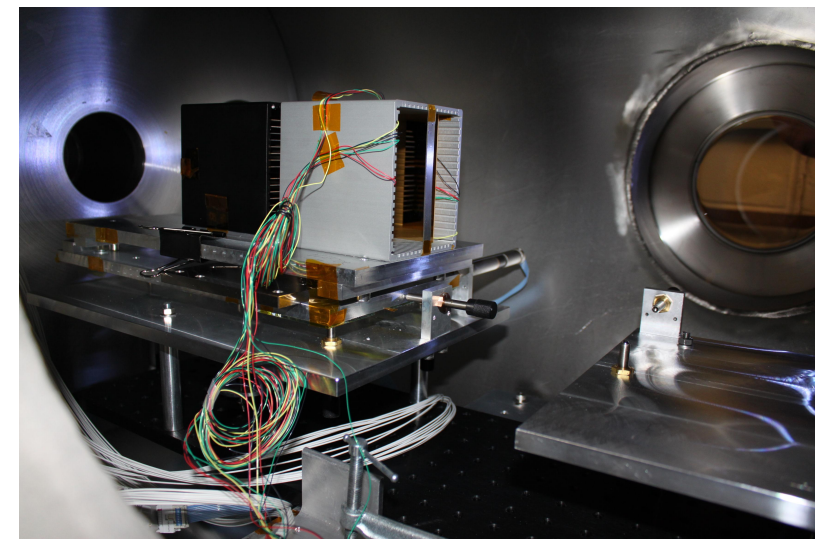

Figure 9. The MFO test module in active arrangement at the X-ray test facility of the University of Colorado at Boulder. The piezo actuators were $2 \mathrm{~mm}$ in diameter, $0,7 \mathrm{~mm}$ in thickness, with maximum bending length of $0.025 \mathrm{~mm}$.

\section{Active Multi Foils X-RAY OptICS}

Multi Foil X-ray Optics can be further improved by applying the principles of active X-ray optics. To exploit this possibility, we performed experiments with adjusting the optical profiles of the surfaces of X-ray optics mirrors and modules with active elements (piezo actuators). These tests were performed both in the laboratory and at the University of Colorado Boulder $\mathrm{X}$-ray facility in the course of full aperture tests in 2012.

One of the modules was tested in an active X-ray approach, adjusting the mirrors/system surfaces with actuators. The X-ray tests confirmed expectations that the application of active X-ray optics principles can further improve the performance of MFO modules. 

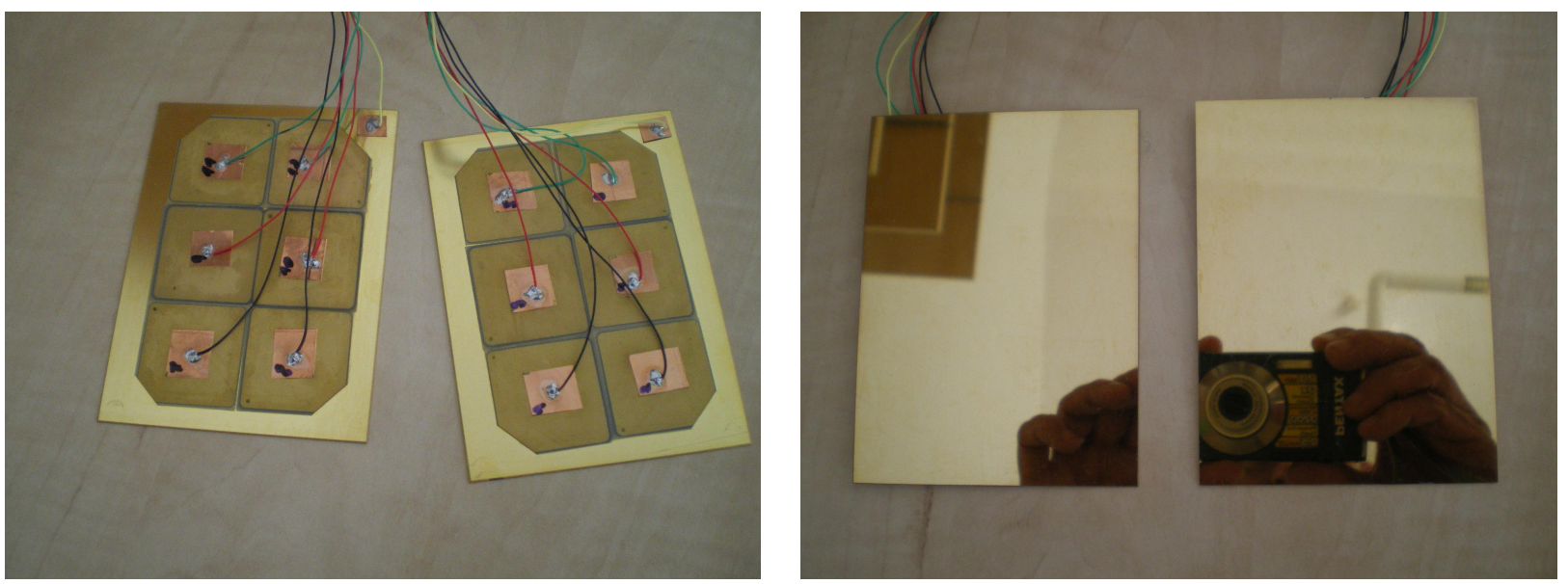

Figure 10. Test specimens of X-ray optics mirrors with glued thin piezo actuators.

\section{Conclusion}

Various innovative designs and technologies for future large X-ray telescopes have been analysed in detail on the basis of extensive research work. Experiments are continuing in an effort to further improve the accuracy and performance of the optics. The KB alternative for astronomical X-ray telescopes was investigated in detail, based on new test modules. The results were quite promising, and justified further efforts in this direction. The important advantage of $\mathrm{KB}$ is its low cost, as there is no need for bending to small curvatures and/or to use expensive mandrels. Less expensive optics can maintain a high effective area even with a reduced budget.

\section{ACKNOWLEDGEMENTS}

We acknowledge the support provided by the Ministry of Education, Youth and Sports of the Czech Republic, in the framework of project ME09028 and ME09004. The investigations related to the ESA IXO/Athena project were supported by ESA PECS project No. 98039. We also acknowledge collaboration with Dr. J. Sik and Dr. M. Lorenc from ON Semiconductor Czech Republic, and with the team of Prof. Webster Cash from the University of Colorado at Boulder for X-ray tests of KB modules in their X-ray facility. VT acknowledges the MEYS ESF Project CZ.1.07/2.3.00/20.0092, under the title "BIOOPT-XUV Research Team Advancement at the Faculty of Biomedical Engineering, Czech Technical University in Prague"

\section{REFERENCES}

[1] Hudec R., Valnicek B., Cervencl J. et al. 1991, SPIE, 1343, 162 .

[2] Hudec R., Pina L., Inneman A. 1999, SPIE, 3766, 62.

[3] Hudec R., Pina L., Inneman A. 2000, SPIE, 4012, 422.

[4] Hudec R., Inneman A., Pina L. 2001, in Lobster-Eye: Novel X-ray Telescopes for the 21st Century, New Century of X-ray Astronomy, ASP Conf. Proc., 251, 542.

[5] Hudec R., Pina L., Inneman A. et al. 2005, SPIE, $5900,276$.

[6] Inneman A., Hudec R., Pina L., Gorenstein P. 1999, SPIE, 3766, 72.

[7] Inneman A., Hudec R., Pina L. 2000, SPIE, 4138, 94.

[8] Kirkpatrick P. and Baez A. V. , J. Opt. Soc. Am. 38, 766 (1948).

[9] Marsch H. et al. 1997, Introduction to Carbon Technologies, University of Alicante.

[10]White, N. E.; Hornschemeier, A. E. 2009, Bulletin of the American Astronomical Society, Vol. 41, p.388.

[11] White, N. E.; Parmar, A.; Kunieda, H.; International X-ray Observatory Team, 2009, Bulletin of the American Astronomical Society, Vol. 41, p.357.

[12] http://ixo.gsfc.nasa.gov

[13]Bavdaz M. et al., 2010, Proceedings of the SPIE, Volume 7732, pp. 77321E-77321E-9.

[14]Zhang W.W. et al. 2010, Proceedings of the SPIE, Volume 7732, pp. 77321G-77321G-8.

[15] Ghigo M. et al. 2010, Proceedings of the SPIE, Volume 7732, pp. 77320C-77320C-12.

[16] http://www .mpe.mpg.de/athena/home.php?lang=en 